\title{
Storia sociale della bicicletta
}

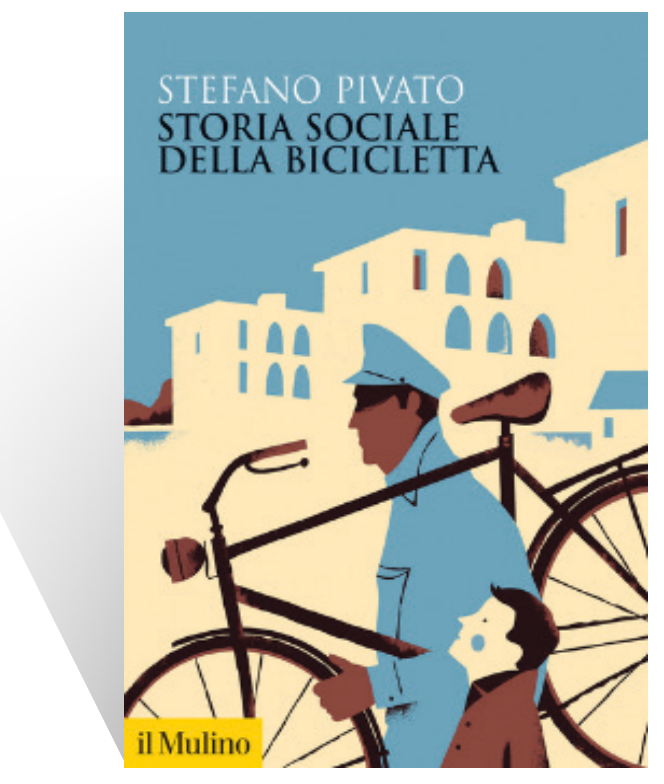

FICHA BIBLIOGRÁFICA

Stefano Pivato, Storia sociale della bicicletta, Bologna, Il Mulino, 2019, 251 págs, ISBN 978-88-15-28521-8

\section{Eleonora Belloni I Università di Siena}

\begin{abstract}
“Traverso le viti di UNA BiCiCletta si può anche scrivere la storia d'Italia”. Con questa citazione di Gianni Brera - uno dei più grandi cantori del ciclismo, e dello sport, in Italia - si apre Storia sociale della bicicletta di Stefano Pivato. Le 251 pagine del volume, organizzato in dieci capitoli tematici, dimostrano che Brera aveva pienamente ragione, e che davvero attraverso la storia della due ruote è possibile ricostruire (e narrare in modo magistrale, nel caso di Pivato) la storia dell'Italia e degli italiani in età contemporanea.

Storia sociale della bicicletta va tra l'altro a colmare un vuoto nella storiografia, italiana e non solo, ben evidenziato dall'A. nelle prime righe dell'Introduzione quando nota come "in una storiografia in gran parte rivolta alla ricostruzione di personalità, battaglie e idee politiche $[. .$.$] la bicicletta non risulta possedere i quarti di nobiltà sufficienti per assurgere a$ oggetto di studio da parte degli storici” (p. 7). Ma anche restringendo il campo alla sola storia
\end{abstract}


della mobilità e dei trasporti, la storia della bicicletta e del "muoversi in bicicletta" è stata non di rado relegata a un ruolo marginale e "introduttivo" a una storia della mobilità che è stata soprattutto storia della mobilità motorizzata, finendo per identificare nel semplice, e in fondo sempre uguale a se stesso, mezzo a due ruote un oggetto reso superato e obsoleto dal mito della motorizzazione a ogni costo.

La ricostruzione di Pivato contribuisce innanzi tutto a sfatare questo mito, dimostrando come la bicicletta non solo abbia accompagnato gli italiani nel lungo e spesso accidentato cammino verso la modernità, ma sia stata essa stessa simbolo e strumento di modernità, di anticonformismo, di vocazione rivoluzionaria votata a spezzare regole, abitudini, status quo consolidati. "Oggetto estraneo all'uomo dell'Ottocento" (p. 12) - tanto che per i primi temerari velocipedisti vengono messi a punto veri e propri prontuari di educazione alla guida del nuovo mezzo - la bicicletta accompagna invece gli italiani nell'ingresso nel nuovo secolo, quello che per il paese segnerà l'avvio del decollo industriale. E non è un caso che l'industria meccanica del ciclo divenga ben presto elemento centrale e trainante di quel take off, con un nucleo produttivo dove ai tanti laboratori artigianali si affiancano ben presto anche industrie di grandi dimensioni (una tra tutte, la ditta Edoardo Bianchi, sorta nel 1885 nella Milano capitale ciclistica del paese e affermatasi poi in tutto il mondo) garantendo al paese l'affrancamento dalla dipendenza estera e, di conseguenza, un abbassamento dei prezzi al consumo che sarà alla base della popolarizzazione del mezzo nelle prime decadi del Novecento.

Nel momento in cui fotografa il cammino dell'Italia verso la modernità, la storia della pratica ciclistica diviene tuttavia anche storia del difficile rapporto del Paese con quella modernità. Un incontro arrivato in ritardo rispetto ad altri paesi, e proprio per questo a lungo rincorso anche a costo di bruciare le tappe o di perdere di vista il vero significato di quel processo. Di questo rapporto controverso con la modernità ci raccontano bene le pagine del volume che ricostruiscono le tante resistenze incontrate dalla bicicletta allinizio del proprio cammino: come sottolinea l'A., "agli entusiasmi dei neofiti delle due ruote si oppongono quanti definiscono il nuovo mezzo 'mostro meccanismo', 'diabolico strumento' o 'macchina infernale”' (p. 29). Dalle autorità municipali ad autorevoli esponenti della scienza medica, dai cattolici ai socialisti, tante furono le voci che pretesero di cogliere i molteplici pericoli (soprattutto per alcune categorie "a rischio" come donne, giovani, preti, ma anche rispettabili professionisti) del lasciarsi irretire dalle promesse di indipendenza e libertà offerte dal mezzo a due ruote. Particolarmente degna di nota la vicenda del rapporto tra donna e bicicletta, cui non a caso l'A. dedica un intero capitolo del suo volume, il quarto. Lo sdegno moralizzatore provocato dalla vista delle prime avventurose signore dell'alta società in sella a una due ruote fu forse pari solamente al fascino che da subito il nuovo mezzo esercitò sull'universo femminile, pronto a cogliere la potenzialità emancipatrice e "liberatrice" di un'innovazione che più di ogni altra avrebbe contribuito, secondo una celebre definizione, a emancipare le donne, liberandole (ma solo dopo un cammino lungo e accidentato) dal peso delle ingombranti crinoline ottocentesche e da quello ancor più ingombrante del maschilismo moralizzatore dell'epoca.

Del rapporto controverso con la modernità testimonia altresì la rapidità con cui il $\mathrm{Pa}$ ese seppe abbandonare l'infatuazione ciclistica a favore di un nuovo amore, quello per il motore. A guardar bene, i divieti municipali di fine Ottocento (a Firenze un regolamento di fine 
secolo ne proibiva la circolazione alle Cascine nelle ore centrali del pomeriggio nel timore che potesse ostacolare il passeggio), quelli emanati dalle autorità repubblichine nei drammatici mesi della Resistenza e quelli riservati alla bicicletta (ma anche ai pedoni) nelle strade della motorizzazione di massa in cui non cè più posto per una mobilità lenta si rivelano facce di una stessa medaglia che identifica nella bicicletta lo strumento di ventate rivoluzionarie da reprimere a difesa dello status quo dominante.

Così come facce della stessa medaglia sono il recupero strumentale della bicicletta operato dal fascismo in chiave autarchica e dai governi dei primi anni Settanta in chiave di austerity. Politiche miopi, improntate al "qui ed ora" e mancanti di qualsiasi lettura di lungo periodo, che hanno contribuito a caratterizzare il difficile e controverso rapporto degli italiani con la bicicletta, costruendovi attorno quell'immagine di povertà e di sacrificio di cui gli italiani, forse, stanno ancora cercando di liberarsi.

Eppure la bicicletta è stata e rimane uno dei pochi elementi di continuità nella costruzione dell'identità nazionale: stanno a dimostrarlo, nel volume, le bellissime pagine ("Biciclette di carta") che ricostruiscono la traccia profonda lasciata dalla bicicletta nella cultura popolare, dalla letteratura ai dialetti, dal cinema alla canzone. O quelle epiche dedicate alla passione per il ciclismo eroico, primo e per lungo tempo unico sport nazionale, capace come pochi di dividere e insieme di unire un paese a più riprese frammentato e lacerato da ferite profonde (tanto è vero che, come sottolinea l'A., il Giro a differenza del Tour fa da specchio alle "Mille Italie" più che a un'unica nazione unita). "Lo sport della bicicletta diviene metafora della velocità di una nazione avviata verso la prima rivoluzione industriale e fa entrare nella lingua italiana un nuovo linguaggio figurato. Il verbo 'pedalare' viene a designare un impegno senza risparmio nella vita e nel lavoro ancor prima che nello sport” (p. 119). Se il duello Coppi-Bartali fotografa e infiamma l'Italia della ricostruzione (ma contribuisce anche - secondo una vulgata popolare alimentata dal giornalismo politico dell'epoca e che l'A. contribuisce a riportare nei giusti ranghi della ricostruzione storica - a preservarla da una nuova ondata rivoluzionaria in occasione dell'attentato a Togliatti nel luglio del 1948), non è un caso che la fine di quella stagione del ciclismo eroico, "macchiato" dapprima dall'ingresso nell'era televisiva e consumistica, poi dagli scandali del doping, coincida con la (apparentemente) definitiva marginalizzazione della bicicletta nell'Italia del miracolo e dell'acquisizione del modello consumistico di massa, che della ricostruzione segna il definitivo superamento.

Se la storia narrata in queste pagine fosse un film, sarebbe suggestivo ipotizzarne il finale con quell'immagine evocativa, ricordata dall'A., delle utilitarie del miracolo che "mettono sul loro tettuccio un modello di bicicletta ripiegabile, la Graziella" (p. 191): con l'avanzata del boom non cè più posto sulle strade italiane per la due ruote (semmai per le due ruote motorizzate, Vespa e Lambretta), "prodotto di un'Italia contadina e provinciale" (p. 191). Un "cambio di testimone", un'inversione di rotta che neppure le esigenze dell'austerity di inizio anni Settanta sarebbero riuscite (perlomeno non in Italia) a capovolgere. Certo, sul primo momento, presi da una rinnovata euforia per la bicicletta, gli italiani si precipitano ad acquistarla mandando in tilt il mercato della due ruote o recuperano i vecchi modelli riposti in soffitta, forse alla ricerca anche "di un passato felice e consolante" (p. 194). Ma si tratta, a ben vedere, di un fuoco paglia: passata l'austerity, le biciclette vengono di nuovo riposte in un angolo e le macchine tornano a invadere le strade cittadine. 
Eppure... le ultime pagine del volume di Pivato ci ricordano che un nuovo umanesimo, ispirato alle esigenze ecologiste e ambientaliste, ma anche a una rinnovata sensibilità alla tutela del territorio e del patrimonio culturale, sembra adesso possibile. La bicicletta, straordinaria macchina del tempo, pare candidarsi a guidare il paese verso un ritorno al futuro all'insegna della rivoluzione dell'antimodernità. 